\title{
Glomerular Filtration Rate Estimation using Cystatin C Levels Identifies Subclinical Renal Dysfunction in Patients with Rheumatoid Arthritis
}

Makoto Fukuda ( $\square$ makotennis@gmail.com )

Saga University

Naoki Sawa

Toranomon Hospital

Hiroki Mizuno

Toranomon Hospital

Daisuke Ikuma

Toranomon Hospital

Rikako Hiramatsu

Toranomon Hospital

Akinari Sekine

Toranomon Hospital

Masahiro Kawada

Toranomon Hospital

Eiko Hasegawa

Toranomon Hospital

Noriko Hayami

Toranomon Hospital

Masayuki Yamanouchi

Toranomon Hospital

Tatsuya Suwabe

Toranomon Hospital

Junichi Hoshino

Toranomon Hospital

Motoaki Miyazono

Saga University

Yoshifumi Ubara

Toranomon Hospital 
Keywords: Cystatin C, eGFR(cre), eGFR(cys), rheumatoid arthritis, activities of daily living(ADL)

Posted Date: November 30th, 2021

DOI: https://doi.org/10.21203/rs.3.rs-1083387/v1

License: (c) (1) This work is licensed under a Creative Commons Attribution 4.0 International License. Read Full License 


\section{Abstract}

\section{Background}

Methotrexate is widely used to treat rheumatoid arthritis (RA) but can cause very serious side effects, including pancytopenia, in patients with renal impairment who have an estimated glomerular filtration rate (GFR) of $<60 \mathrm{ml} / \mathrm{min} / 1.73 \mathrm{~m}^{2}$. In patients with low muscle volume such as elderly patients, GFR can be measured as higher value when calculated using serum creatinine [eGFR(cre)], so more accurate estimation using cystatin C [eGFR(cys)] is preferred.

Method

We evaluated 173 patients with RA who visited Toranomon Hospital in 2019 for factors that may contribute to the difference between eGFR(cre) and eGFR(cys) [eGFR(cre-cys)]. Activities of daily living (ADL) (walking, 1 point; using a cane, 2 points; using a wheelchair, 3 points) was added as a parameter.

Results

In univariate analysis using Spearman's rank correlation coefficient, eGFR(cre-cys) was negatively correlated with body weight, height, body surface area (BSA), subcutaneous fat area (SFA)/BSA, albumin, and creatinine kinase, and positively correlated with age, erythrocyte sedimentation rate (ESR), and urinary protein. eGFR(cre-cys) was higher in patients who used a cane or wheelchair than those who could walk unaided. Multiple regression analysis showed that ADL and age contribute significantly to eGFR(cre-cys).

Conclusion

Patients using a cane or wheelchair are susceptible to the higher eGFR(cre-cys) values and overestimation of eGFR(cre), resulting in failed detect subclinical renal dysfunction. For such patients, eGFR(cys) should be evaluated to prevent serious side effects of methotrexate that are easily developed on patients with renal dysfunction.

\section{Introduction}

Estimates of glomerular filtration rate (GFR) using serum creatinine [eGFR(cre)] are frequently inaccurate, leading to over- or underestimation of renal function. Serum creatinine level is greatly influenced by muscle mass, and [eGFR(cre)] is estimated to be higher in patients with low muscle mass such as those who have been lying in bed for a long time. Since serum cystatin $C$ is not affected by muscle mass, cystatin $C$ measurement is particularly useful in such cases where [eGFR(cre)] is considered unreliable. A method to estimate GFR using cystatin C [eGFR(cys)] has been proposed by Inker et al, in which eGFR(cys) was calculated by CKD-EPI cystatin C equation. They proposed that the combined creatininecystatin $\mathrm{C}$ equation performed better than equations based on either of these markers alone and may be useful as a confirmatory test for chronic kidney disease ${ }^{(1)}$. Horio et al proposed that for Japanese 
patients, it is better to use the "Eq cys+nonrenal" (2). Targońska-Stepniak et al. reported that in patients with rheumatoid arthritis (RA), cystatin C (CysC) may be an accurate indicator of GFR as well as a marker of intensity of chronic inflammatory processes ${ }^{(3)}$. Cystatin $\mathrm{C}$ concentration has been reported to be significantly higher in patients of RA with high disease activity, and eGFR(cys) became lower ${ }^{(3)}$. Methotrexate is now the most popular drug worldwide for the treatment of RA, but it can cause lifethreatening side effects, including pancytopenia, in patients with renal impairment who have an eGFR of $<60 \mathrm{ml} / \mathrm{min} / 1.73 \mathrm{~m}^{2}$. Therefore, accurate evaluation of eGFR is needed ${ }^{(4)}$. However, calculating eGFR using serum creatinine [eGFR(cre)] can result in inaccurate renal function estimates, leading to overdiagnosis of chronic kidney disease. The clinical reasons for the difference between eGFR(cre) and eGFR(cys) in patients with RA remain unknown. Here, we aim to clarify them.

\section{Materials And Methods}

\section{Patients}

This was a retrospective, single-center cohort study. Out of 258 patients with RA who visited Toranomon Hospital for treatment between July (2019) 1 and September (2019) 30 , and met a criteria of RA ${ }^{(4)}$ at this consultation, 173 agreed to providing their clinical information and were enrolled in this study.

\section{Clinical information}

The following clinical information from blood and urine samples was collected from all patients at their latest consultation: serum creatinine $(\mathrm{mg} / \mathrm{dL})$, serum CysC $(\mathrm{mg} / \mathrm{L})$, age (years), gender, height $(\mathrm{cm})$, body weight $(\mathrm{kg})$, body mass index $(\mathrm{BMI})\left(\mathrm{kg} / \mathrm{m}^{2}\right)$, body surface area (BSA; Du Bois method) $\left(\mathrm{m}^{2}\right)$, disease duration (years), serum albumin $(\mathrm{g} / \mathrm{dL})$, creatine kinase $(\mathrm{U} / \mathrm{L})$, urea nitrogen $(\mathrm{mg} / \mathrm{dL})$, triglycerides $(\mathrm{mg} / \mathrm{dL})$, total cholesterol $(\mathrm{mg} / \mathrm{dL})$, C-reactive protein $(\mathrm{mg} / \mathrm{dL})$, erythrocyte sedimentation rate (ESR) $(\mathrm{mm} / \mathrm{h})$, hemoglobin $(\mathrm{g} / \mathrm{dL})$, urinary protein $(\mathrm{g} /$ day), history of diabetes mellitus, history of hypertension, corticosteroid use, methotrexate use, and biological drug use. eGFR(cre) ${ }^{(6)}$ and eGFR(cys) ${ }^{(1)}$ (both in $\mathrm{mL} / \mathrm{min} / 1.73 \mathrm{~m}^{2}$ ) were measured using previously reported methods, and [eGFR(cre-cys)] was obtained by subtracting eGFR(cys) from eGFR(cre).

Although it is difficult to assess muscle mass quantitatively and objectively, it is not unreasonable to assume that muscle mass corresponds to activities of daily living (ADL) in daily clinical practice. Therefore we used the following indicators; walking, 1 point (consistent with stage 1 according to Steinbrocker classification); using a cane, 2 points (consistent with stage 2 according to Steinbrocker classification); using a wheelchair, 3 points( consistent with stage 3 qnd 4 according to Steinbrocker classification). Subcutaneous fat area $\left(\mathrm{cm}^{2}\right)$ /BSA was measured at the level of the iliac superior border, by using synapse Vincent software ${ }^{(7)}$.

The procedures followed were in accordance with the Declaration of Helsinki and its revisions, and this study was approved by the local research ethics board (approval number: 2062-B). 


\section{Statistical analysis}

Analyses were performed using the EZR software package (ver. 3.5.2). Continuous variables were expressed as the median and interquartile range (IQR) according to their distribution. P values were calculated using Spearman's rank correlation coefficient for continuous variables, Mann-Whitney U test for nominal variables, and Kruskal-Wallis test and Bonferroni correction for comparison between the three groups. All $P$ values were two sided, and $P<0.05$ was considered statistically significant.

\section{Results}

\section{Patient characteristics}

In this cohort, most RA patients are in good condition with low C-reactive protein and normal serum albumin, because of treatment by using biologic agents and/or methotrexate. This study is an evaluation in patients whose disease activity due to RA has been adequately controlled by treatment. Out of total 173 patients, 136 patients (78.6\%) were ADL 1 (walk), 32 patients (18.5\%) were ADL 2(cane), and 5 patients (2.9\%) were ADL3(wheel chair)(Table 1).

eGFR(cre-cys) was calculated to determine the cases in which eGFR(cre) had been overestimated.

Significant correlations were confirmed between serum creatinine and eGFR(cre) ( $P<0.001)$, eGFR(cys) $(P<0.001)$, and eGFR(cre-cys) $(P<0.01)$ (Fig. 1a). Similarly, there was a significant correlation between age and eGFR(cre), eGFR(cys), and eGFR(cre-cys) ( $P<0.001$ for all) (Fig. 1b).

\section{Risk factors contributing to a higher eGFR(cre-cys) in all patients}

In a univariate analysis using Spearman's rank correlation coefficient, eGFR(cre-cys) showed a significant negative correlation with body weight, height, BSA, subcutaneous fat area (SFA)/BSA, albumin and creatinine kinase $(P<0.001)$ (Table 1). eGFR(cre-cys) showed a significant positive correlation with age, ESR, and urinary protein $(P<0.001)$ (Table 1). No significant differences in eGFR(cre-cys) were observed between genders, duration of RA, corticosteroid use, or biological drug use (Table 1). The Kruskal-Wallis test showed that eGFR(cre-cys) was higher in patients who used a cane (group ADL2) $(P<0.001)$ or a wheelchair (group ADL3) $(P<0.001)$ than in those who could walk unaided (group ADL1). The Bonferroni test revealed a significant difference in eGFR(cre-cys) between ADL1 and ADL2 ( $P<0.001)$ and between ADL1 and ADL3 ( $P=0.049$ ), indicating that eGFR(cre-cys) was correlated with ADL (Fig. 1c).

We then performed a multiple regression analysis using eGFR (cre-cys) as the objective variable, and multiple factors (including ADL, age, serum albumin, biological drug use, BMI, C-reactive protein, serum creatine kinase, female gender, methotrexate, and urinary protein, that a univariate analysis showed 
significant difference) as explanatory variables. ADL and age showed important risk factors contributing to a high eGFR(cre-cys) (Table 2).

\section{Characteristic of 20 patients predicting the highest eGFR(cre-cys)}

Out of total 173 patients, the characteristic of 20 patients predicting highest eGFR(cre-cys) was analysed.

20 patients were sorted in order of highest eGFR(Cre -Cys)(Table 3-1), and showed max difference of 58.4 (case 1) to minimum difference of 13.1 (case 20) between eGFR(Cre-Cys). 20 patients included ADL $1(n=5), A D L 2(n=12)$ and ADL3(n=3).15 patients showed lower ADL patients (ADL1+ADL2), and included 14 patients who received or are scheduled to receive knee or hip surgery because of severe knee and hip arthritis. 5 patients showed lower BMI less than 18.5,2 patients showed higher BMI over 25, and 13 patients showed normal range BMI.9 patients showed $80 \mathrm{~s}, 6$ patients showed 70 s, and 5 patients showed 60 s and younger (Table 3-1).

20 patients were sorted in order of lowest eGFR(cys) (Table 3-2). Out of 20 patients, two patients had eGFR(cre) $<60$, but 12 patients had eGFR(cys) <60; of these 12 patients, 3 had a lower BMI $(<18.5)$ and lower SFA $\left(<30.4 \mathrm{~cm}^{2} / \mathrm{mm}^{2}\right)$ indicating lower muscle mass. 8 patients had a BMI within the normal range $(18.5-25)$ but very higher SFA $\left(>50 \mathrm{~cm}^{2} / \mathrm{mm}^{2}\right)$ indicating lower muscle mass (Table 3$)$. This indicates that patients with lower $A D L$ have higher risk of adverse effects from methotrexate might be overlooked when using eGFR(cre), but that eGFR(cys) may accurately detect the presence of this risk.

\section{Discussion}

Laterza et al. reported that cysC levels in the blood are independent of age and gender, and that cysC is superior to creatinine for detecting impaired GFR ${ }^{(8)}$. Kazama et al. reported that age, gender, glucose tolerance, proteinuria, systemic inflammation, lupus, or systemic use of steroids did not interfere in the relationship between sodium thiosulfate clearance and 1/CysC, and that serum CysC measurement is an excellent diagnostic test for identifying patients with subclinical renal dysfunction ${ }^{(9)}$. Sato et al. reported that serum CysC $>1.09 \mathrm{mg} / \mathrm{L}$ can identify a lower GFR more accurately than serum creatinine, with serum CysC $>1.365 \mathrm{mg} / \mathrm{L}$ strongly suggesting a low GFR in patients who have RA with secondary amyloidosis (10). To date, little attention has been paid to RA patients with renal dysfunction because they have low serum creatinine levels so their eGFR(cre) appears normal, but according to Kimura et al., serum CysC, which is unaffected by muscle quantity, is a potentially superior marker to creatinine for estimating renal function ${ }^{(11)}$. In patients with muscular dystrophy, eGFRcys measurements were in strong agreement (differences $<20 \%$ ) with values of GFR simultaneously measured by inulin renal clearance ${ }^{(11)}$.

In conclusion, our study indicates that RA patients with lower ADL scores (those using a cane or wheelchair) are susceptible to the higher eGFR(cre-cys) values and overestimation of eGFR(cre), resulting 
in a failure to identify those with subclinical renal dysfunction. For such patients, eGFR(cys) should be used instead to prevent serious side effects from methotrexate that are easily developed on patients with renal dysfunction.

Serum creatinine level is greatly influenced by muscle mass, and [eGFR(cre)] is estimated to be higher in patients with low muscle mass. Since serum cystatin $C$ is not affected by muscle mass, cystatin $C$ measurement is particularly useful in cases where [eGFR(cre)] is considered unreliable.

\section{Limitation}

In order to show the usefulness of eGFR(cys) on patients with RA, it was more desirable to measure eGFR(in) using inulin clearance in addition to eGFR(Cre) and eGFR(cys), and to compare the measured

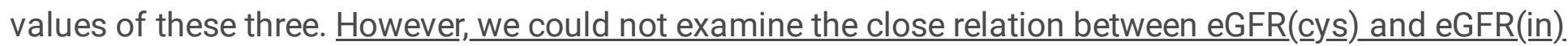
by using inulin in routine clinical practice. Therefore we tested only the difference between eGFR(cre) and eGFR(cys) on patients with RA. This study is data from a single center, and more data collection is desired for generalization. Although the titer of cystatin $\mathrm{C}$ could be subject to the effects of thyroid hormone, we did not examine thyroid hormone.

\section{Declarations}

Disclosures

The authors declare no competing financial interests. The authors also declare that they have no conflicts of interest. All patients gave written informed consent.

Acknowledgement

This study was funded by the Okinaka Memorial Institute for Medical Research.

registration number:20620-B

\section{References}

1. Inker, L.A., Schmid, C.H., Tighiouart, H. et al. Estimating glomerular filtration rate from serum creatinine and cystatin C. N Engl J Med. 2012; 367: 20-29

2. Horio M, Imai E, Yasuda Y, Watanabe T, Matsuo S; Collaborators Developing the Japanese Equation for Estimated GFR. GFR estimation using standardized serum cystatin C in Japan. Am J Kidney Dis. 2013 Feb;61(2):197-20

3. Targońska-Stepniak B, Majdan M. Cystatin C concentration is correlated with disease activity in rheumatoid arthritis patients. Scand J Rheumatol. 2011;40(5):341-6.

4. Rheumatoid Arthritis Clinical Trial Archive Group. The effect of age and renal function on the efficacy and toxicity of methotrexate in rheumatoid arthritis. J Rheumatol. 1995 Feb;22(2):218-23. 
5. Aletaha D, Neogi T, Silman AJ, Funovits J, Felson DT, Bingham CO 3rd, et al. 2010 Rheumatoid arthritis classification criteria: an American College of Rheumatology/European League Against Rheumatism collaborative initiative. Arthritis Rheum. 2010 Sep;62(9):2569-81.

6. Matsuo S, Imai E, Horio M, Yasuda Y, Tomita K, Nitta K, et al. on behalf of the collaborators developing the Japanese equa- tion for estimating GFR. Revised Equations for estimating glomerular filtration rate(GFR)from serum creatinine in Japan. Am J Kidney Dis 53: 982-992, 2009.

7. Ohshima S. Volume analyzer SYNAPSE VINCENT for liver analysis. J Hepatobiliary Pancreat Sci. 2014 Apr;21(4):235-238.

8. Laterza OF, Price CP, Scott MG. Cystatin C: an improved estimator of glomerular filtration rate? Clin Chem. 2002 May;48(5):699-707. Review.

9. Kazama JJ, Kutsuwada K, Ataka K, Maruyama H, Gejyo F. Serum cystatin C reliably detects renal dysfunction in patients with various renal diseases. Nephron. 2002 May;91(1):13-20.

10. Sato H, Kuroda T, Tanabe N, Ajiro J, Wada Y, Murakami S, et al. Cystatin C is a sensitive marker for detecting a reduced glomerular filtration rate when assessing chronic kidney disease in patients with rheumatoid arthritis and secondary amyloidosis. Scand J Rheumatol. 2010;39(1):33-7.

11. Kimura K, Morita H, Daimon M, Horio M, Kawata T, Nakao T, et al. Utility of Cystatin C for Estimating Glomerular Filtration Rate in Patients With Muscular Dystrophy. Int Heart J. 2016 May 25;57(3):3868.

\section{Tables}

Due to technical limitations, table 1, 2 and 3 is only available as a download in the Supplemental Files section.

\section{Figures}



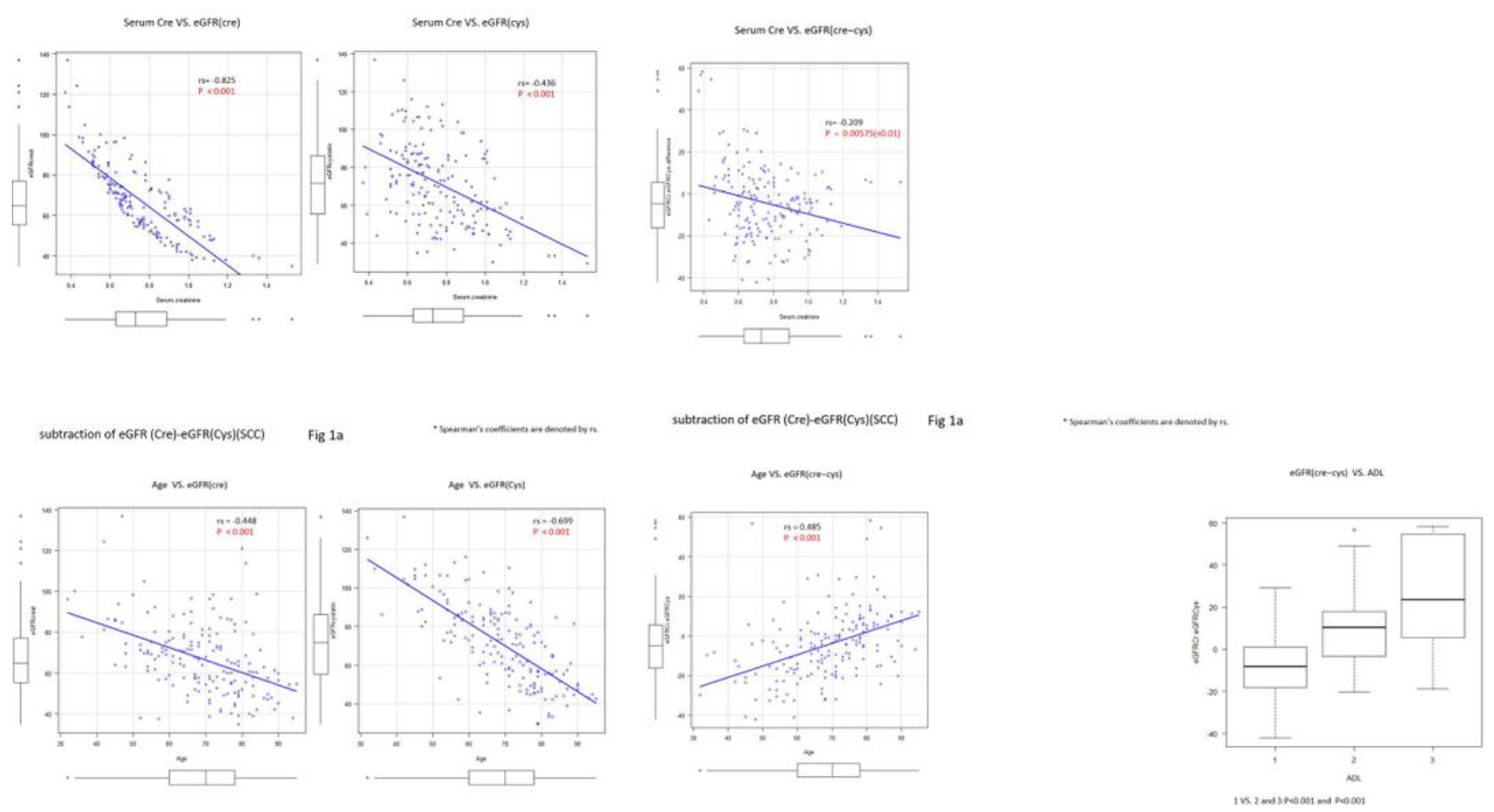

Fig 1b

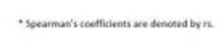

Fig 1b

Fig 1c

\section{Figure 1}

(a-c) Spearman's Rank correlation coefficient. (a) serum creatinine correlated with eGFR(cre), eGFR(cys) and eGFR(cre-cys). serum cystatin C correlated with eGFR(cre-cys). (b) Age correlated with eGFR(cre), eGFR(cys), and eGFR(cre-cys). (c) Box plot using Kruskal-Wallis test. ADL2 and ADL3 are significantly higher in eGFR(cre-cys) than ADL1.

\section{Supplementary Files}

This is a list of supplementary files associated with this preprint. Click to download.

- Table1.xlsx

- Table2.xlsx

- Table31.xlsx

- Table32.xlsx 